\title{
LOBULITE LINFOCITÁRIA ESCLEROSANTE EM PACIENTES COM DIABETES MELLITUS TIPO 1
}

\author{
SCLEROSING LYMPHOCYTIC LOBULITIS IN PATIENTS WITH TYPE 1 DIABETES MELLITUS
}

Alfredo Ribeiro-Silva

Docente. Departamento de Patologia. Faculdade de Medicina de Ribeirão Preto - USP

CorResPondÊncIA: Prof. Dr. Alfredo Ribeiro-Silva. Laboratório de Patologia Ginecológica e Mamária. Departamento de Patologia. Faculdade de Medicina de Ribeirão Preto - USP. Av. Bandeirantes, 3900 - Campus Universitário Monte Alegre; CEP 14049-900, Ribeirão Preto - SP, Brasil. E-mail:_arsilva@fmrp.usp.br / FAX: 1636331068 - Fone: 1636023119

Ribeiro-Silva A. Lobulite linfocitária esclerosante em pacientes com diabetes mellitus tipo 1. Medicina (Ribeirão Preto) 2005; 38 (3/4): 290-293.

RESUMO: Modelo do estudo: Revisão da literatura

Importância do problema: A mastopatia diabética é uma doença mamária benigna freqüentemente associada ao diabetes melitus do tipo 1 de longa evolução.

Comentários: Embora consagrado pelo uso, o termo mastopatia diabética não é correto porque um quadro morfológico idêntico (lobulite linfocítica esclerosante) pode aparecer em mulheres com outras doenças autoimunes e mesmo em mulheres sem nenhuma alteração sistêmica aparente.

Descritores: Mama. Diabetes Mellitus. Doença Fibrocística da Mama.

\section{1- INTRODUÇÃO}

A mastopatia diabética é uma doença mamária benigna frequientemente associada ao diabetes melitus do tipo $1^{1}$. Vários nomes já foram propostos para essa condição: doença fibrosa da mama do diabetes do tipo $1^{2}$, mastopatia do diabetes insulino dependente ${ }^{3}$, doença mamária fibrosa diabética ${ }^{4}$, mastite linfocítica com fibrose $^{5}$, mastopatia fibrosa diabética ${ }^{6}$ e mastopatia diabética ${ }^{7}$. O termo lobulite linfocitária esclerosante é o mais aceito atualmente porque, conforme será discutido adiante, nem todas as pacientes que apresentam essas lesões mamárias são diabéticas ${ }^{1,8}$.

\section{2- CARACTERÍSTICAS CLÍNICAS}

Clinicamente a mastopatia diabética é caracterizada por lesões solitárias ou múltiplas em uma ou ambas as mamas, de consistência endurecida, indolor, de limites bem definidos e móveis. O diâmetro dessas massas varia de poucos milímetros a mais de $6 \mathrm{~cm}$. Geralmente é de localização subareolar, mas pode aparecer em qualquer parte da mama. Na maioria das vezes os nódulos requerem excisão cirúrgica, mas regressões espontâneas já foram relatadas ${ }^{9}$. Após a excisão o nódulo pode recorrer no mesmo local, em outro local da mesma mama ou na mama contralateral. A taxa de recorrência é de $30 \%{ }^{10}$.

As pacientes geralmente são jovens e muitas delas, porém nem todas, apresentam diabetes do tipo 1 de longa duração. Por outro lado, cerca de $70 \%$ das pacientes com diabetes do tipo 1 desenvolvem a doença ${ }^{8}$. Nesses casos, normalmente várias complicações da diabete já estão presentes na época da detecção do nódulo mamário, como retinopatia diabética, neuropatia e nefropatia ${ }^{11}$. Lesões similares podem 
aparecer em homens com diabetes melitus do tipo 1 de longa evolução, e são consideradas uma forma especial de ginecomastia ${ }^{5}$. A mastopatia diabética não está associada ao diabetes do tipo $2^{8}$.

No exame mamográficos as lesões aparecem como áreas densas assimétricas, podendo haver espessamento da pele subjacente ${ }^{12}$. No exame de ultra-som as áreas palpáveis aparecem como massas hipoecóicas de margens indistintas e caracteristicamente com acentuada sombra acústica ${ }^{7}$. A ressonância magnética não é utilizada para esses casos.

\section{3- CARACTERÍSTICAS PATO- LÓGICAS}

Macroscopicamente os nódulos são firmes e esbranquiçados, de limites precisos, e medem em média de 2 a $6 \mathrm{~cm}$ no maior eixo. Aos cortes, a superfície é constituída por um tecido homogêneo de coloração branco-acinzentada que pode ser difícil de ser distinguido do tecido fibroso que circunda o tecido mamário adjacente.

Devido à baixa celularidade, tanto a punção biópsia aspirativa (PBA) quanto a biópsia por agulha grossa ("core biopsy") não são bons métodos para o diagnóstico, resultando em material inadequado em mais de $50 \%$ dos casos $^{4}$. Quando representativos, os esfregaços obtidos através da PBA apresentam escassos agrupamentos de células epiteliais em meio a linfócitos e a fibroblastos epitelióides, caracterizados por células isoladas com citoplasma abundante e núcleos vesiculosos, algumas vezes binucleados. O fundo é constituído por tecido conjuntivo denso e hialinizado $^{13}$.

O quadro histológico é constituído por fibrose densa associada a infiltrado inflamatório linfocítico predominantemente perilobular (Figuras 1 e 2). $\mathrm{O}$ estroma fibroso apresenta fragmentos de colágeno semelhantes ao observado na quelóide, entremeados por fibroblastos epitelióides (Figura 3). O infiltrado linfocítico é constituído por células $\mathrm{B}^{10}$. A Tabela I resume os achados histológicos.

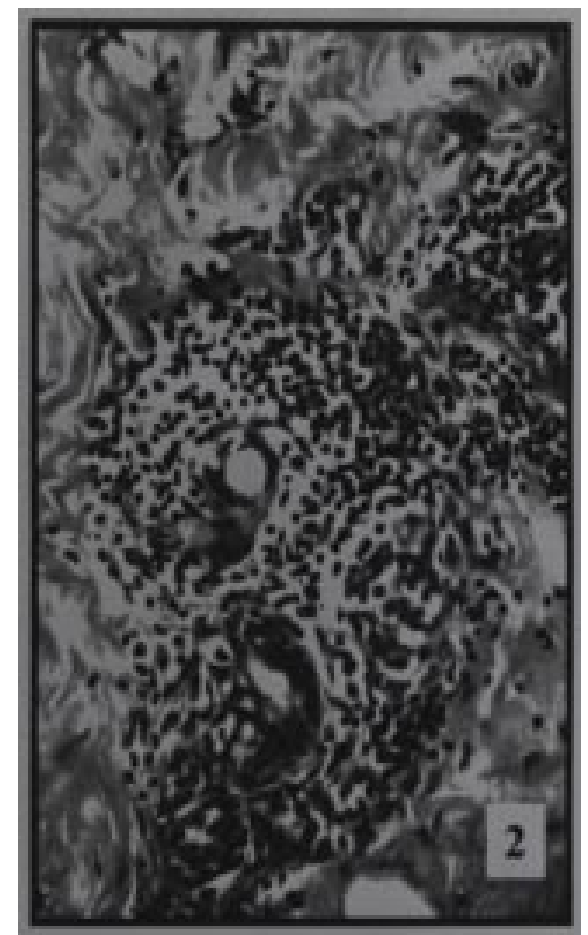

Figura 2. Detalhe de lóbulo mamário demonstrando que o infiltrado inflamatório é predominantemente linfocítico (Hematoxilina e eosina, x200). 


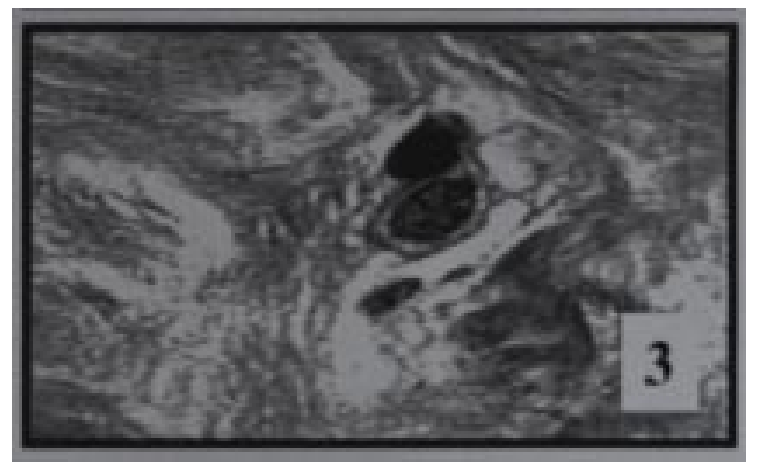

Figura 3. Fibroblastos epitelióides de permeio às fibras de colágeno (Hematoxilina e eosina, x400).

\section{4- DIAGNÓSTICO DIFERENCIAL}

O aspecto histopatológico é bastante característico e não há necessidade de técnicas especiais para o diagnóstico. Entre os diagnósticos diferenciais destacam-se as fibroses mamárias inespecíficas, os fibroadenomas com acentuada fibrose, a fibromatose mamária, os leiomiomas, os tumores desmóide, os pseudolinfomas e linfomas mamários, e as mastites ${ }^{1}$. A presença dos fibroblastos epitelióides e o infiltrado linfocítico de células B diferencia a lobulite linfocítica esclerosante de outras formas de mastite ${ }^{14}$.

\section{5- PATOGÊNESE}

A patogênese da lobulite linfocítica esclerosante é desconhecida. Sabe-se, entretanto, que existe uma forte associação dessa patologia com o diabetes do tipo 1, doenças tireoidianas, e doenças do tecido con-

\section{Tabela I: Características histológicas da mastopatia diabética.}

1. Exuberante fibrose do estroma interlobular

2. Infiltrado inflamatório linfocítico predominantemente perilobular

3. Discreto infiltra do inflamatório linfocítico perivascular e periductal

\section{Atrofia lobular}

5. Fibroblastos epitelióides em meio a fibras de colágeno fragmentadas

juntivo. Esses dados sugerem que a lobulite linfocítica esclerosante seja uma manifestação de uma doença sistêmica autoimune. Entretanto, em um estudo com oito pacientes apresentando o quadro histopatológico típico da lobulite linfocítica esclerosante, Williams e colaboradores verificaram que quatro delas não apresentavam nem diabetes, nem doenças auto-imunes, nem qualquer outra doença sistêmica ${ }^{15}$.

\section{6- CONCLUSÃO}

Existe uma forte associação entre a lobulite linfocítica esclerosante e o diabetes melitus do tipo 1 , de tal maneira que essa patologia também é conhecida como mastopatia diabética. Esse termo, entretanto, não é correto porque um quadro morfológico idêntico (lobulite linfocítica esclerosante) pode aparecer em mulheres com outras doenças autoimunes e mesmo em mulheres sem nenhuma alteração sistêmica aparente.

Ribeiro-Silva A. Sclerosing lymphocytic lobulitis in patients with type 1 diabetes mellitus. Medicina (Ribeirão Preto) 2005; 38 (3/4): 290-293.

Abstract: Design of the study: Review of the literature

Relevance: Diabetic mastopathy is a benign mammary disease frequently associated with a long term type 1 diabetes mellitus.

Comments: Although worldwide used, the term diabetic mastopathy is not correct because an identical morphological pattern (sclerosing lymphocytic lobulitis) may be seen in women with other autoimmune disorders and even in women with no apparent systemic disturb.

Keywords: Breast. Diabetes Mellitus. Fibrocystic Disease of Breast. 


\section{REFERÊNCIAS BIBLIOGRÁFICAS}

1 - Haj M, Weiss M, Herskovits T. Diabetic sclerosing lymphocytic lobulitis of the breast. J Diabetes Complications 2004; 18: $187-91$.

2 - Soler NG, Khardori R. Fibrous disease of the breast, thyroiditis, and cheiroarthropathy in type I diabetes mellitus. Lancet 1984; 1: 193-5.

3 - Byrd BF Jr, Hartmann WH, Graham LS, Hogle HH. Mastopathy in insulin-dependent diabetics. Ann Surg 1987; 205: 52932.

4 - Logan WW, Hoffman NY. Diabetic fibrous breast disease. Radiology 1989; 172: 667-70.

5 - Hunfeld KP, Bassler R. Lymphocytic mastitis and fibrosis of the breast in long-standing insulin-dependent diabetics. A histopathologic study on diabetic mastopathy and report of ten cases. Gen Diagn Pathol 1997; 143: 49-58.

6 - Shaffrey JK, Askin FB, Gatewood OM, Brem R. Diabetic Fibrous Mastopathy: Case Reports and Radiologic-Pathologic Correlation. Breast J 2000; 6: 414-7.

7 - Wong KT, Tse GM, Yang WT. Ultrasound and MR imaging of diabetic mastopathy. Clin Radiol 2002; 57: 730-5.

8 - Kudva YC, Reynolds C, O'Brien T, Powell C, Oberg AL, Crotty TB. "Diabetic mastopathy," or sclerosing lymphocytic lobulitis, is strongly associated with type 1 diabetes. Diabetes Care 2002; 25: 121-6.
9 - Bayer U, Horn LC, Schulz HG. Bilateral, tumorlike diabetic mastopathy-progression and regression of the disease during 5-year follow up. Eur J Radiol 1998; 26: 248-53.

10 - Ely KA, Tse G, Simpson JF, Clarfeld R, Page DL. Diabetic mastopathy. A clinicopathologic review. Am J Clin Pathol 2000; 113: $541-5$.

11 - Pluchinotta AM, Talenti E, Lodovichetti G, Tiso E, Biral M. Diabetic fibrous breast disease: a clinical entity that mimics cancer. Eur J Surg Oncol 1995; 21: 207-9.

12 - Andrews-Tang D, Diamond AB, Rogers L, Butler D. Diabetic mastopathy: adjunctive use of ultrasound and utility of core biopsy in diagnosis. Breast J 2000; 6: 183-8.

13 - Peppoloni L, Buttaro FM, Cristallini EG. Diabetic mastopathy. A report of two cases diagnosed by aspiration cytology. Acta Cytol 1997; 41: 1349-52.

14 - Seidman JD, Schnaper LA, Phillips LE. Mastopathy in insulinrequiring diabetes mellitus. Hum Pathol 1994; 25: 819-24.

15 - Williams PH, Rubin CM, Theaker JM. Sclerosing lymphocytic lobulitis of the breast. Clin Radiol 1995; 50: 165-7.

Recebido em 11/11/2005

Aprovado em 30/12/2005 